\title{
Childhood and Adolescence Vitiligo: Clinicoepidemiological Profile and Its Impact on Quality of Life
}

\author{
Nermin Karaosmanoğlu' (, Pınar Özdemir C̣etinkaya² (1), Ișıl Göğem İmren' (1), Esra Kıratlı Nalbant' (D, \\ Engin Karaaslan ${ }^{3}$ (i)
}

'Department of Dermatology and Venereology, Ankara Training and Research Hospital, Ankara, Turkey

${ }^{2}$ Department of Dermatology and Venereology, Nazilli State Hospital, Aydın, Turkey

${ }^{3}$ Department of Dermatology and Venereology Nusaybin State Hospital, Mardin, Turkey

ORCID IDs of the authors: N.K. 0000-0002-3462-I628; P.Ö.C.. 0000-000I-5286-3260; i.G.i. 0000-0002-9574-323I; E.K.N. 0000-0002-II5402I9; E.K. 0000-0003-29II-5082.

Cite this article as: Karaosmanoğlu N, C̣etinkaya PÖ, İmren IG, Nalbant EK, Karaaslan E. Childhood and Adolescence Vitiligo: Clinicoepidemiological Profile and Its Impact on Quality of Life. Cyprus J Med Sci 2020; 5(I): 4I-6.

\section{BACKGROUND/AIMS}

Vitiligo is an acquired, idiopathic, generally progressive pigmentation disorder of an unknown etiology. Vitiligo usually begins during childhood or adolescence. This study aimed to evaluate the clinical and demographic characteristics of this disease as well as understand its impact on patient quality of life (QoL).

\section{MATERIAL and METHODS}

Thirty-one vitiligo patients aged 5-16 years who visited the outpatient dermatology clinic were included in the study. The demographic information of all the patients was collected. A complete clinical examination was performed, and the disease-related features were recorded. Moreover, all the patients completed the children's dermatology life quality index (CDLQI).

\section{RESULTS}

Of the 31 patients, 20 were girls $(64.5 \%)$, and II were boys (35.5\%). The mean patient age was $10.33 \pm 4.19$ years. Vitiligo vulgaris was the most common (51.6\%) clinical type of vitiligo. The most commonly involved areas were the trunk (54.9\%), face (54.9\%), and extremities (4I.7\%). In the study group, $32.3 \%$ of the patients (I0 of 31 ) had an accompanying autoimmune disease. The median CDLQI score of the patient group was 6 (min-max: 0-22). There was a significant correlation between patient age and CDLQ| score $(r=0.369, p=0.04 \mid)$.

\section{CONCLUSION}

$\checkmark$ itiligo has a different clinicoepidemiological profile in children and adolescents. This disease significantly lowers the patient's QoL. Our study found a significant correlation between patient age and CDLQI score. In clinical practice, it is important to remember that vitiligo is not only a cosmetic disorder; it may negatively affect multiple aspects of the patient's QoL.

Keywords: Epidemiology, quality of life, vitiligo

\section{INTRODUCTION}

Vitiligo is generally an acquired, progressive pigmentation disorder of an unknown etiology $(\mathrm{l}, 2)$. The onset of vitiligo is usually during childhood or adolescence (3), with the prevalence being $0.04 \%-2.2 \%$ in children (2). The disease does not cause a physical disturbance; however, its clinical manifestations may have negative psychosocial effects and decrease the patient's quality of life (QoL), especially when the onset is during childhood or adolescence (I).

The Children's Dermatology Life Quality Index (CDLQI) is used for children aged 5-I6 years and evaluates the influence of skin diseases on the QoL considering the findings during last week. It was first created by Lewis-Jones and Finlay (4). Balcı et al. (5) then translated the scale into the Turkish language and conducted validation studies.

This index has been used for many childhood dermatological diseases (6); however, few studies have used this index for vitiligo (I, 2,7-II). Limited information is available about the influence of vitiligo on the QoL of children; therefore, we decided to conduct research on this topic with the patients managed at the outpatient clinic. 
This study aimed to evaluate the clinical and demographic characteristics of the disease and the effects on the QoL of children and adolescents.

\section{MATERIALS and METHODS}

Total 3I vitiligo patients aged 5-16 years who visited the outpatient dermatology clinic were included in the study. The research protocol was approved by the institutional ethics committee. All the participants and their parents were informed about the study, and informed consent form was obtained.

Diagnoses were established by dermatologists in charge of the study and were based on the clinical symptoms. Demographic details of all the patients, including the age, sex, body mass index, age of onset, duration of disease, presence of associated autoimmune and endocrine diseases, family history, and story of previous treatments were recorded. The patients were questioned about the daily duration of sun exposure, use of sunscreen, seasonal exacerbation, as well as symptoms, such as pruritus and erythema. A complete clinical examination was performed and information about the sites, percentage of the body surface area involved, presence of leukotrichia and Koebner phenomenon was recorded from the clinic notes. The status of the disease was defined as active and stable. If there was emergence of new lesions and/or enlargement in the size of existing lesions during the previous 3 months, the disease was recorded as active. The standard working classification system was used to determine the clinical types of vitiligo, and the patients were divided into the following six groups: focal, segmental, vulgaris, acrofacial, mucosal, and universal (I2).

The I0-question CDLQI is often used in dermatology practice to assess how a skin disease has affected a patient's daily life activities during the previous week. The validated CDLQI was the instrument mainly used in this study. It has a Likert scale scored from 0-3 ( $0=$ not at all, I=only a little, $2=$ quite a lot, and $3=$ very much) and consists of 7 parts that help evaluate different characteristics as follows: "symptoms/feelings," "leisure," "school/ holidays," "daily activities," "personal relationships," "sleep," and "treatment." The maximum possible score was 30 and the low-

\section{Main Points:}

- Vitiligo does not cause a physical disturbance; however, its clinical manifestations may have negative psychosocial effects and decrease the patient's quality of life, especially when the onset is during childhood or adolescence.

- Of the 31 patients, 20 were girls (64.5\%), and II were boys (35.5\%). There is a female preponderance in the present study.

- Ten (32.3\%) patients had accompanying autoimmune and endocrine diseases such as autoimmune tiroiditis, celiac disease, alopecia areata, type I diabetes mellitus, and halo nevus.

- The median children's dermatology life quality index (CDLQI) score of the patient group was 6 (range: 0-22). There was a significant correlation between patient age and CDLQI scores.

- Vitiligo is common in children and adolescents and has a different clinicoepidemiological profile. est possible score was 0. Higher the score, lower the QoL (4). The children completed the CDLQI themselves. Few younger children could not read or write; for these children, the parents were asked to read out the questions and record their answers without directing the children in their point of view.

\section{Statistical Analyses}

All the analyses were performed using the Statistical Package for the Social Sciences, version 16.00 (SPSS Inc., Chicago, USA) and a p value $<0.05$ was considered statistically significant. Normality of the data was tested. The qualitative variables were described as absolute and relative frequencies. Mean and standard deviation values were calculated for normal quantitative variables, while median and minimum-maximum values were calculated for the non-normal variables. Non-normally distributed quantitative variables were compared using the Mann-Whitney $U$ test in the presence of two variables and the Kruskal Wallis test in the presence of more than two variables; Spearman's Rho analyses was used for correlations between two variables.

\section{RESULTS}

Of the 3 I patients, 20 were girls (64.5\%), and II were boys (35.5\%). The mean patient age was $10.33 \pm 4.19$ years. Twenty patients were children ( $<12$ years old) and II were adolescent (12-16 years old).

The mean body mass index was $20.62 \pm 3.50 \mathrm{~kg} / \mathrm{m}^{2}$. The average age at disease onset was $8.89 \pm 3.84$ years. The median disease duration was 12 months (range: I-72) (Table I).

Eleven patients $(35.5 \%)$ had focal, $16(51.6 \%)$ had vulgaris, 2 (6.5\%) had acrofacial, and $2(6.5 \%)$ had segmental vitiligo. Nobody had mucosal or universal types of vitiligo (Table 2 ). Most patients (17, 54.9\%) had lesions on the trunk; further, 17 (54.9\%) had lesions on the face (Figure I). The detailed distribution of the lesions is presented in Figure 2. The percentage of the involved body surface area is shown in Figure 3. Twenty-two (71\%) patients complained of itching, and 27 (87.1\%) had erythema. Eight (25.8\%) reported seasonal exacerbation of the disease. Twenty-two patients (71\%) had an active and progressive disease course, while $9(29 \%)$ had a stable course. Eight patients (25.8\%) had a positive Koebner phenomenon. Leukotrichia was found in 6 (19.4\%) of the patients. None had a mucosal lesion (Table 3).

Total 23 patients ( $74.2 \%$ ) had a sun exposure of $<5$ hours per day, $5(16.1 \%)$ had $5-10$ hours of exposure, and $3(9.7 \%)$ had $>10$ hours of exposure per day. Of the 31 patients, only 4 (I2.9\%) reported using sunscreen regularly.

Twenty-one (67.7\%) patients had no accompanying autoimmune and endocrine diseases, while $4(12.9 \%)$ had autoimmune tiroiditis, 2 (6.5\%) had celiac disease, 2 (6.5\%) had alopecia areata, I (3.2\%) had type I diabetes mellitus, and I (3.2\%) had halo nevus. Seven patients $(22.6 \%)$ had a family history of vitiligo. Seventeen patients $(54.8 \%)$ had not undergone any treatment previously, while $5(16.1 \%)$ used topical steroids, $3(9.7 \%)$ used topical pimecrolimus, $3(9.7 \%)$ used topical tacrolimus, and 3 (9.7\%) underwent narrow-band ultraviolet UVB therapy. 

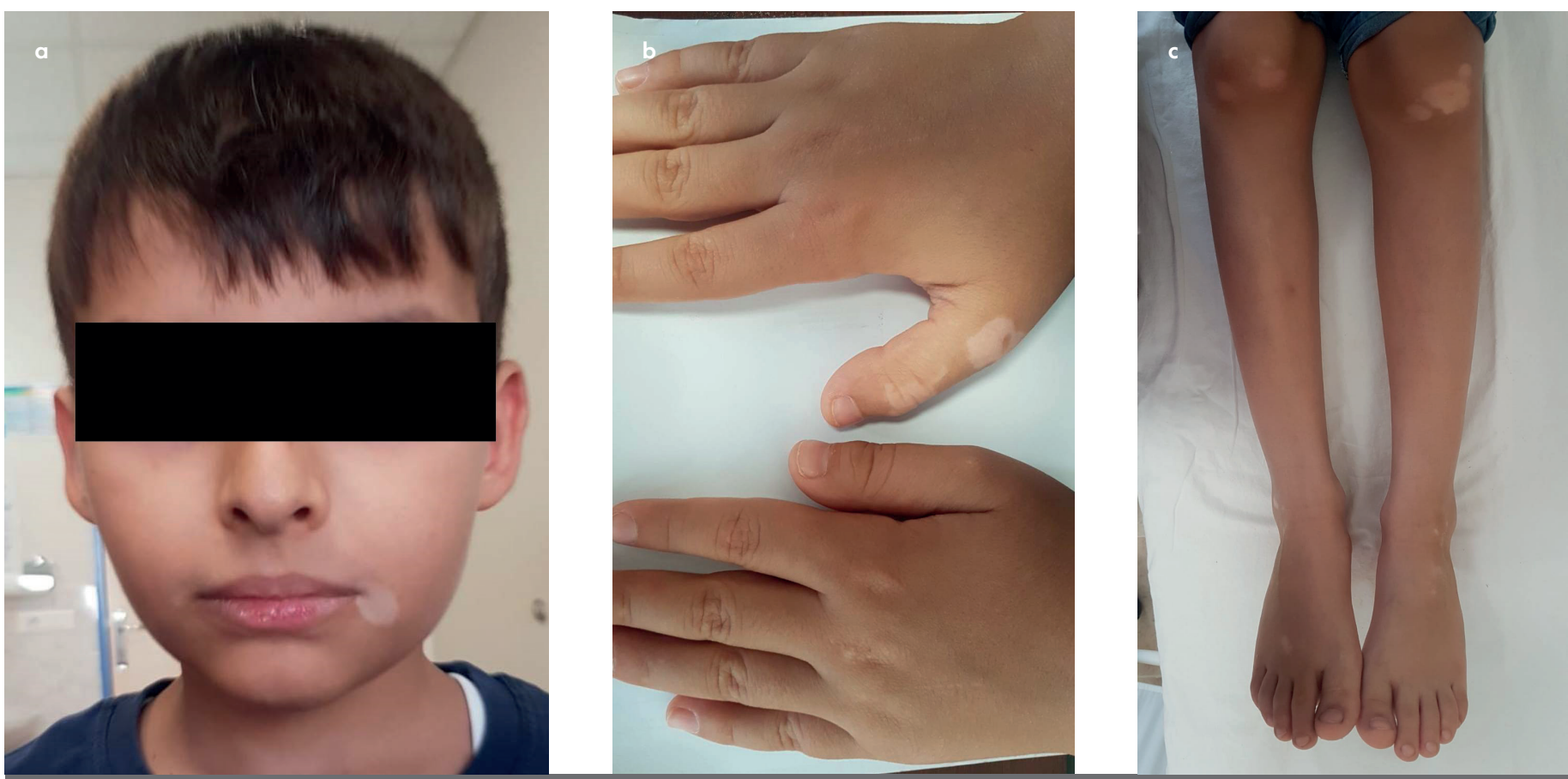

FIGURE I. a-c. Depigmented macules and patches involving the face, hands, knees and feet in a patient with vitiligo vulgaris. (a) Face involvement. (b) Hand involvement. (c) Knee and feet involvement

TABLE I. Demographic characteristics of the vitiligo patients

\section{Demographic characteristics}

Sex

Age

Body Mass Index

Age at Disease Onset

Duration of the Disease

Max: Maximum, Min: Minimum, SD: Standard deviation

\section{Distrubution of the lesions}

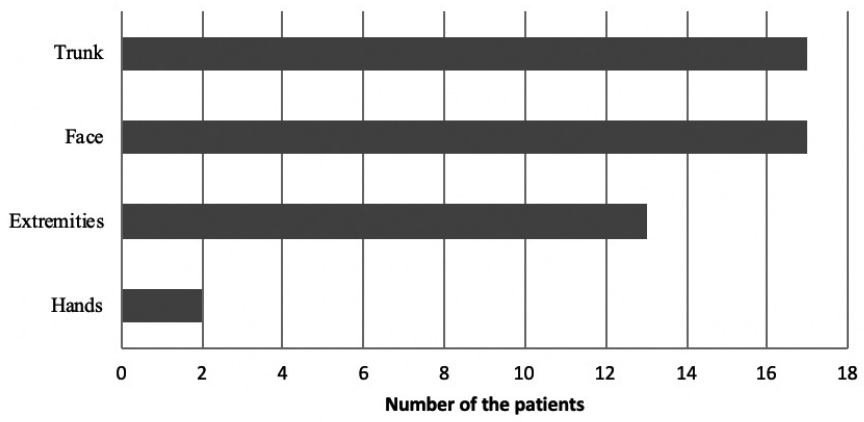

FIGURE 2. Distribution of the lesions

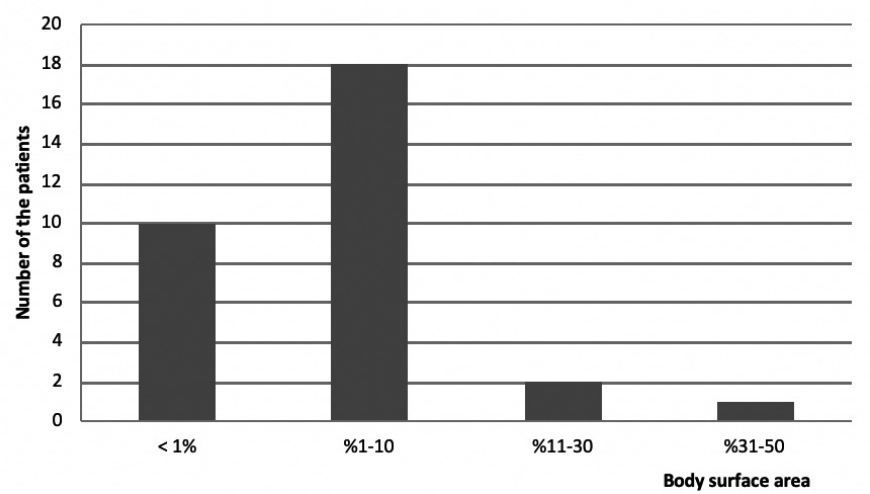

FIGURE 3. The percentage of the involved body surface area 
TABLE 2. Distribution of the types of vitiligo among the patients

\begin{tabular}{|lcc|}
\hline Types of Vitiligo & $\mathbf{n}$ & $\%$ \\
\hline Focal Vitiligo & ॥ & 35.5 \\
Vitiligo Vulgaris & 16 & 51.6 \\
Acrofacial Vitiligo & 2 & 6.5 \\
Segmental Vitiligo & 2 & 6.5 \\
Mucosal Vitiligo & - & - \\
Universal Vitiligo & - & - \\
\hline
\end{tabular}

\begin{tabular}{|lcc|}
\hline TABLE 3. Clinical features of the vitiligo patients & \\
\hline Clinical Features & Present $(\mathrm{n} / \%)$ & Absent $(\mathrm{n} / \%)$ \\
\hline Itching & $22(71)$ & $9(29)$ \\
Erythema & $27(87.1)$ & $4(12.9)$ \\
Seasonal exacerbation & $8(25.8)$ & $23(74.2)$ \\
Active and progressive course & $22(71)$ & $9(29)$ \\
Koebner phenomenon & $8(25.8)$ & $23(74.2)$ \\
Leukotrichia & $6(19.4)$ & $25(80.6)$ \\
\hline
\end{tabular}

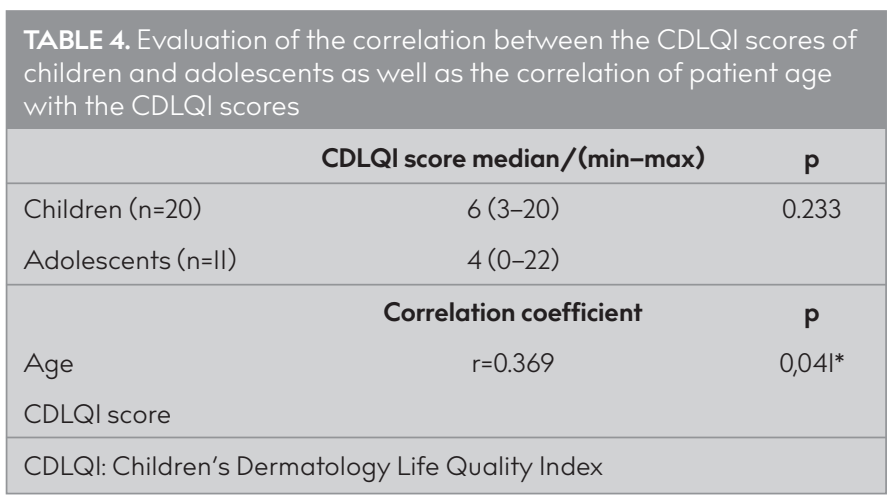

\section{Effect of vitiligo on the CDLQI scores}

The median CDLQI score of the patient group was 6 (range: 0-22). There was no significant difference in the CDLQI scores of boys and girls $(p=0.917)$ as well as those of children and adolescents $(p=0.233)$. There was a significant correlation between patient age and CDLQI scores ( $r=0.369, p=0.041$ ) (Table 4). As the age of the patients increased, the CDLQI scores also increased, and thus, the QoL decreased. There was no significant correlation between age at disease onset and the CDLQI scores ( $p=0.123$ ). There was no significant difference in the CDLQI scores as per vitiligo subtypes $(p=0.350)$. There was no significant correlation between disease duration and the CDLQI scores $(p=0.137)$ or between the percentage of the involved body surface area and the CDLQI scores $(p=0.32 I)$.

\section{DISCUSSION}

$\checkmark$ itiligo is the most commonly encountered disease among disorders of depigmentation. It usually starts during childhood or early adulthood (3). This study aimed to describe the clinical spectrum and epidemiological data of vitiligo in children and adolescents. Moreover, we attempted to determine the effect of vitiligo on their QoL, using the CDLQI.

In $>60 \%$ of the patients in our study group, the age of disease onset was $4-12$ years (mean age: $8.89 \pm 3.84$ years). In 10 out of the 31 patients $(32.3 \%)$, the disease developed at 4-8 years of age, and in $10(32.3 \%)$ subjects, the first symptoms of the disease occurred at 9-12 years of age. The reported disease onset varied greatly across studies; however, most authors have concluded that vitiligo is acquired in the early stages of life, especially at 4-12 years of age (13). When the age at presentation was studied, the mean age was $10.33 \pm 4.19$ years, and most patients (35.5\%) were 9-12 years old. This disparity and delay between the age of onset and age of presentation shows the progressive course of the disease and the need for long-term treatment and follow-up.

A female preponderance of ( $63 \%$ vs. $57 \%$ ) in childhood vitiligo has been reported by most studies, in accordance with our results that showed a higher incidence $(64.5 \%)$ of vitiligo in girls (14-16). However, some other studies have reported an almost equal incidence for boys and girls $(17,18)$. The higher incidence in girls could be attributed to overrepresentation in childhood and adolescence because of the cosmetically disfiguring, de-pigmenting nature of the disease.

In agreement with previous reported $(3,14)$, vitiligo vulgaris was the most common (51.6\%) clinical type of vitiligo in children and adolescents in our study. Focal vitiligo was the second most common (35.5\%) presentation in this study similar to the result published by Halder et al (14). Segmental type of vitiligo is a special clinical type for children because it has been reported more frequently in children. In most studies, the frequency is $>16 \%$, ranging from $4.6 \%-32.5 \%$ (14-18). However, in our patients, segmental vitiligo accounted for only $6.5 \%$ of all cases. We did not observe universal vitiligo, mucosal vitiligo, or mucosal involvement related to other clinical types of vitiligo in our patient group.

The three most commonly involved areas were the trunk (54.9\%), face $(54.9 \%)$ and extremities (41.7\%). In the present study, $22.6 \%$ of the patients had a family history of vitiligo. Various studies have reported that patients with vitiligo have higher rates of positive family history, ranging from $3.3 \%-35 \%(3,14,17,19,20)$.

The incidence of leukotrichia was $19.4 \%$ in the present study. In the literature, the incidence of leukotrichia is known to range from $3.7 \%-25 \%(3,14,19)$. The Koebner phenomenon was present in $25.8 \%$ of our patients, similar to that reported in a previous trial (19).

Vitiligo may be associated with several endocrinopathies and other autoimmune disorders; however, in various studies, the frequency of autoimmune disorders in vitiligo patients was less than that observed in our study (2I-23). In our study group, 32.3\% of the patients $(10 / 31)$ had an accompanying autoimmune disease. Four (12.9\%) had autoimmune tiroiditis, 2 (6.5\%) had celiac disease, 2 (6.5\%) had alopecia areata, I (3.2\%) had type I diabetes mellitus, and I (3.2\%) had halo nevus.

Handa et al. (15) reported an incidence of $1.3 \%$ (7/625) for autoimmune disorders in children with vitiligo. Jaisankar et al (3) reported that none of their patients was diagnosed with autoimmune disorders, while Halder et al. (14) reported that $2.4 \%$ of their patients had alopecia areata (2/82). Schallreuter et al. (23) reported that all of the diseases thought to be associated with 
vitiligo were coincidental except for thyroid diseases and vitiligo patients were susceptible to thyroid diseases withimpaired thyroidal function tests. Alopecia areata is frequently reported in children with vitiligo, with the rate ranging from $0.32 \%-2.4 \%$. Thus, our rate of $6.5 \%$ was higher than that reported in the literature $(14,15)$.

Vitiligo is a disorder of skin color that causes emotional and functional deterioration that leads to a decreased QoL. In the present study, the median CDLQI score of the patient group was 6 (range: 0-22). The QoL was found to be severely impaired in $19.35 \%(n=6)$ of the subjects (scores $>10)$. The literature includes several studies that have assessed the dermatology life quality index in children and adolescents with vitiligo. Dertlioğlu et al (I) evaluated the CDLQI scores in vitiligo patients, atopic dermatitis patients, and controls. The CDLQI scores were significantly elevated in the vitiligo patient group (mean \pm standard deviation: $11.68 \pm 6.54$ ) compared to that in the atopic dermatitis group $(7.74 \pm 4.59)$ and control group (0.62 \pm 0.77$)$. Kruger et al (2) evaluated 74 patients, including children and adolescents, and a control group to compare their disease-related QoL scores. They reported that there was no significant severe impairment in the QoL in most patients. However, the mean score of CDLQI in the patient group was 2.8 (median: 1.5) and that in the control group was 0.6 (median: 0.0), representing a statistically significant difference $(p=0.004)$. Silverberg et al (II) found that the median CDLQI score was 3.0 (interquartile range 5.0) in children and adolescent subjects with vitiligo. Furthermore, Lewis-Jones and Finlay (4) investigated the effect of cutaneous diseases of childhood on the CDLQI scores in a group of pediatric patients to find that the mean CDLQI scores were $5.4 \pm 5.0$ in psoriasis patients, $7.7 \pm 5.6$ in atopic dermatitis patients, and $5.6 \pm 3.8$ in vitiligo patients. Although the scores varied across the studies, they usually remained $<10$. Thus, vitiligo usually lowers the QoL in children and adolescents significantly; however, but usually does not cause a severe impact.

In this study, neither disease duration nor percentage of the involved body surface area influenced the CDLQI scores. Although there was no significant difference in the CDLQI scores of children and adolescents, there was a significant correlation between patient age and CDLQI scores. Thus, as patient age increased, the CDLQI scores increased and the QoL decreased. This finding was consistent with that reported by Silverberg et al (II) who reported that the QoL in adolescents with a disease onset age of 10 years or older further deteriorated, suggesting that disease onset during the developmental stages of ego causes the greatest QoL impairment. Adolescence is a developmental period that includes physical, social, emotional, and cognitive development. Owing to the physical changes associated with puberty, adolescent patients were assumed to be more concerned with their physical symptoms. Thus, it was understandable that the quality of life decreased with disturbing dermatological findings that appeared on their bodies during this process of establishing a sense of identity. In more detail, the psychological findings in vitiligo can be explained by the interaction of cytokines and neuropeptides at the cellular level. Vitiligo is assumed to be an autoimmune disease. However, in the recent years, the concept of psycho-neuro-endocrine-immune system has been established. This system defines a bidirectional connection among stress, neuropeptides, cytokines, and the skin and claims that alterations in the levels of various cytokines and neuropeptides result in development of skin diseases, including vitiligo and neuropsychiatric findings (24).

In conclusion, this study presents the sociodemographic and clinical characteristics of patients with vitiligo aged $<16$ years old from Turkey as well as the effects of this disease on the patient QoL. Our study reveals that vitiligo is common in children and adolescents and has a different clinicoepidemiological profile. There is a female preponderance in the present study. Vitiligo vulgaris was the most common clinical type of vitiligo. Frequency of associated autoimmune disorders is more common. The median CDLQI score of the patients was 6 (range: 0-22), and there was a significant correlation between patient age and CDLQI score. In clinical practice, it is important to remember that vitiligo is not just a cosmetic disorder; it also has a considerable impact on multiple aspects of the patient's QoL.

Ethics Committee Approval: Ethics committee approval was received for this study from Instutional Ethics Committe of Ankara Training and Research Hospital Approval No: 4/2019 Date: 30.05.2019

Informed Consent: Written informed consent was obtained from patients and patients' parents who participated in this study.

Peer-review: Externally peer-reviewed.

Author contributions: Design - N.K., P.Ö.Ç., E.K.N.; Supervision - I.G.I., E.K.; Resource - N.K.; Materials - E.K.N., I.G.I.; Data Collection and/or Processing - E.K., I.G.I, P.Ö.C.., E.K.N., N.K.; Analysis and/or Interpretation - N.K., P.Ö.C.., E.K.; Literature Search - N.K., P.Ö.C.; Writing - N.K., P.Ö.C.; Critical Reviews - N.K., P.Ö.C,, E.K.N., I.G.I., E.K.

Conflict of Interest: The authors have no conflicts of interest to declare.

Financial Disclosure: The authors declared that this study has received no financial support.

\section{REFERENCES}

I. Dertlioglu SB, Cicek D, Balci DD, Halisdemir N. Dermatology life quality index scores in children with vitiligo: comparison with atopic dermatitis and healthy control subjects. Int J Dermatol 2013; 52(I): 96-I0I. [CrossRef]

2. Kruger C, Panske A, Schallreuter KU. Disease-related behavioral patterns and experiences affect quality of life in children and adolescents with vitiligo. Int J Dermatol 2014; 53(I): 43-50. [CrossRef]

3. Jaisankar TJ, Baruah MC, Garg BR. Vitiligo in children. Int J Dermatol 1992; 3I(9): 62I-3. [CrossRef]

4. Lewis-Jones MS, Finlay AY. The Children's Dermatology Life Quality Index (CDLQI): initial validation and practical use. Br J Dermatol 1995; 132(6): 942-9. [CrossRef]

5. Balcı DD, Sangun O, Inandı T. Cross-validation of the Turkish version of the children's dermatology life quality index. J Turk Acad Dermatol 2007; I(4): I-5.

6. Olsen JR, Gallacher J, Finlay AY, Piguet V, Francis NA. Quality of life impact of childhood skin conditions measured using the Children's Dermatology Life Quality Index (CDLQI): a meta-analysis. Br J Dermatol 2016; 174(4): 853-6I. [CrossRef]

7. Schwartz R, Sepulveda JE, Quintana T. [Possible role of psychological and environmental factors in the genesis of childhood vitiligo]. Rev Med Chil 2009; 137(I): 53-62. [CrossRef] 
8. Agarwal S, Ramam M, Sharma VK, Khandpur S, Pal H, Pandey RM. A randomized placebo-controlled double-blind study of levamisole in the treatment of limited and slowly spreading vitiligo. $\mathrm{Br} \mathrm{J}$ Dermatol 2005; 153(I): 163-6. [CrossRef]

9. Bilgic O, Bilgic A, Akis HK, Eskioglu F, Kilic EZ. Depression, anxiety and health-related quality of life in children and adolescents with vitiligo. Clin Exp Dermatol 20Il; 36(4): 360-5. [CrossRef]

10. Njoo MD, Bos JD, Westerhof W. Treatment of generalized vitiligo in children with narrow-band (TL-OI) UVB radiation therapy. J Am Acad Dermatol 2000; 42(2 P+ I): 245-53. [CrossRef]

II. Silverberg JI, Silverberg NB. Quality of life impairment in children and adolescents with vitiligo. Pediatr Dermatol 20I4; 3I(3): 309-I8. [CrossRef]

12. Kovacs SO. Vitiligo. J Am Acad Dermatol 1998; 38(5 Pt I): 647-66; quiz 67-8. [CrossRef]

13. Halder RM. Childhood vitiligo. Clin Dermatol 1997; 15(6): 899-906. [CrossRef]

14. Halder RM, Grimes PE, Cowan CA, Enterline JA, Chakrabarti SG, Kenney JA, Jr. Childhood vitiligo. J Am Acad Dermatol 1987; 16(5 Pt I): 948-54. [CrossRef]

15. Handa S, Dogra S. Epidemiology of childhood vitiligo: a study of 625 patients from north India. Pediatr Dermatol 2003; 20(3): 207-I0. [CrossRef]

16. Pajvani U, Ahmad N, Wiley A, Levy RM, Kundu R, Mancini AJ, et al. The relationship between family medical history and childhood vitiligo. J Am Acad Dermatol 2006; 55(2): 238-44. [CrossRef]
17. Cho S, Kang HC, Hahm JH. Characteristics of vitiligo in Korean children. Pediatr Dermatol 2000; 17(3): 189-93. [CrossRef]

18. Hu Z, Liu JB, Ma SS, Yang S, Zhang XJ. Profile of childhood vitiligo in China: an analysis of 541 patients. Pediatr Dermatol 2006; 23(2): II4-6. [CrossRef]

19. Agarwal S, Gupta S, Ojha A, Sinha R. Childhood vitiligo: clinicoepidemiologic profile of 268 children from the Kumaun region of Uttarakhand, India. Pediatr Dermatol 2013; 30(3): 348-53. [CrossRef]

20. Nicolaidou E, Antoniou C, Miniati A, Lagogianni E, Matekovits A, Stratigos $A$, et al. Childhood- and later-onset vitiligo have diverse epidemiologic and clinical characteristics. J Am Acad Dermatol 2012; 66(6): 954-8. [CrossRef]

21. Dawber RP. Clinical associations of vitiligo. Postgrad Med J 1970; 46(535): 276-7. [CrossRef]

22. Palit A, Inamadar AC. Childhood vitiligo. Indian J Dermatol Venereol Leprol 2012; 78(I): 30-4I. [CrossRef]

23. Schallreuter $K U$, Lemke $R$, Brandt $O$, Schwartz $R$, Westhofen $M$, Montz R, et al. Vitiligo and other diseases: coexistence or true association? Hamburg study on 321 patients. Dermatology 1994; 188(4): 269-75. [CrossRef]

24. Lotti T, Zanardelli M, D'Erme AM. Vitiligo: what's new in the psycho-neuro-endocrine-immune connection and related treatments. Wien Med Wochenschr 2014; 164(I3-14): 278-85. [CrossRef] 